\title{
Cardiovascular Pathology: A Subspecialty of Surgical Pathology
}

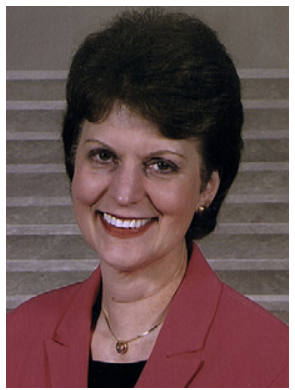

Gayle L. Winters, MD

Guest Editor

For hundreds of years, anatomists, physiologists, and physicians studied the cardiovascular system at autopsy. Cardiovascular pathologists were autopsy pathologists. Within only the last 50 years, the advent of cardiopulmonary bypass, cardiovascular surgical techniques, endomyocardial biopsy, and cardiac transplantation has brought cardiovascular specimens to the surgical pathology bench. These specimens range in size from $0.2 \mathrm{~cm}$ (endomyocardial biopsy) to the entire heart (explant). Compared to other areas of surgical pathology, cardiovascular pathology is a "new" subspecialty. I can remember opening a surgical pathology specimen container in 1984 and seeing an entire heart for the first time.

Unfortunately, many surgical pathologists did not embrace the arrival of cardiovascular specimens. Cardiovascular pathology, for the most part, is not tumor pathology. It requires knowledge of cardiac anatomy, cardiac physiology, cardiology, and cardiac surgical procedures. In fact, in some European countries, it is common for cardiovascular pathologists to be trained in clinical cardiology. The increasing importance of making a diagnosis on cardiovascular specimens from living patients put pressure on surgical pathology divisions to find someone to deal with these specimens. Cardiovascular diagnoses could now impact living patients. Additional pressure came during the 1980s from the widely expanding cardiac transplant programs that insisted on cardiac pathology expertise. In more recent years, cardiovascular pathologists have not only had to deal with cardiovascular tissue itself, but with the results of interventional procedures-we must examine stents, pacemakers and defibrillators, prosthetic valves, and ventricular-assist devices. In addition, rapidly growing knowledge in the area of molecular pathology has played an everexpanding role in how we process tissue and classify cardiovascular diseases.

Cardiovascular pathology is a non-boarded subspecialty of pathology. Although a few cardiovascular pathology fellowships are available, many cardiovascular pathologists practicing today are self-taught. In 1985, cardiovascular pathologists formed the Society for Cardiovascular Pathology (SCVP), which has approximately 200 members worldwide. Cardiovascular Pathology, the official journal of the Society, was first published in 1992. The primary goals of SCVP are educational and collegial since many cardiovascular pathologists are the sole person in that specialty within their department. It is important that surgical pathologists recognize their limitations in dealing with cardiovascular specimens, particularly those not commonly encountered, and seek consultation from cardiovascular pathologists with more expertise in the specific area.

In this volume of Surgical Pathology Clinics devoted to Cardiovascular Pathology, we attempt to provide useful, practical information on the majority of cardiovascular specimens that may arrive on the surgical bench-valves (native and prosthetic), endomyocardial biopsies (native and transplant), blood vessels ranging from large (aorta) to small, cardiac tumors, and an approach to the explanted heart and cardiovascular devices. In the tradition of this series, each article is replete with photos and has summary boxes 
containing key features, differential diagnoses, and pitfalls.

I am indebted to all my coauthors, who have provided their time and expertise. My special thanks to our two cardiac pathology fellows, Michael Seidman and Paul VanderLaan, who have each taken on first authorship of a chapter while performing all of their "routine" duties and preparing for Pathology Boards. Thanks to $\mathrm{Dr}$ John Goldblum, Consulting Editor, and Elsevier for remembering to include Cardiovascular Pathology in the Surgical Pathology Clinics series and Joanne Husovski of Elsevier for her patience, encouragement, and support.

Gayle L. Winters, MD Department of Pathology Brigham and Women's Hospital

Harvard Medical School

75 Francis Street Boston, MA 02115, USA

E-mail address: gwinters@partners.org

\section{DEDICATION}

This issue is dedicated to my Dad for many years of guidance, encouragement, and support. 